\title{
Thin-Branching Papillae Present
}

National Cancer Institute

\section{Source}

National Cancer Institute. Thin-Branching Papillae Present. NCI Thesaurus. Code 695513.

A morphologic architectural pattern indicating the presence of papillary structures

forming thin branches. 